\title{
Schwannoma of the Orbit
}

\author{
Kwang Seog Kim¹, \\ Jin Woo Jung ${ }^{1}$, \\ Kyung Chul Yoon', \\ Yu Jin Kwon', \\ Jae Ha Hwang', \\ Sam Yong Lee ${ }^{1}$ \\ Departments of ${ }^{1}$ Plastic and Reconstructive \\ Surgery and ${ }^{2}$ Ophthalmology, Chonnam \\ National University Medical School, \\ Gwangju, Korea
}

No potential conflict of interest relevant to this article was reported.

\begin{abstract}
Background: A schwannoma is a benign, slow-growing peripheral nerve sheath tumor that originates from Schwann cells. Orbital schwannomas are rare, accounting for only $1 \%$ of all orbital neoplasms. In this study, we retrospectively review orbital schwannomas and characterize clinical, radiologic, and histologic features of this rare entity.

Methods: A retrospective review was performed to identify patients with histologically confirmed orbital schwannoma, among a list of 437 patients who had visited our hospital with soft tissue masses within the orbit as the primary presentation between 2010 and 2014. Patient charts and medical records were reviewed for demographic information, relevant medical and family history, physical examination findings relating to ocular and extraocular sensorimotor function, operative details, postoperative complications, pathologic report, and recurrence.

Results: Five patients $(5 / 437,1.1 \%)$ were identified as having histologically confirmed orbital schwannoma and underwent complete excision. Both computed tomography (CT) and magnetic resonance imaging (MRI) studies were not consistent in predicting histologic diagnosis. There were no complications, and none of the patients experienced significant scar formation. In two cases, patients exhibited a mild postoperative numbness of the forehead, but the patients demonstrated full recovery of sensation within 3 months after the operation. None of the five patients have experienced recurrence.

Conclusion: Orbital schwannomas are relatively rare tumors. Preoperative diagnosis is difficult because of its variable presentation and location. Appropriate early assessment of orbital tumors by CT or MRI and prompt management is warranted to prevent the development of severe complications. Therefore, orbital schwannomas should be considered in the differential diagnosis of slow-growing orbital masses.
\end{abstract}

Keywords: Schwann cells / Neurilemmoma / Orbital neoplasm / Surgery

\section{INTRODUCTION}

Schwannomas are slow-growing benign peripheral nerve sheath tumors that originate from Schwann cells. Orbital schwannomas are rare, accounting for only $1 \%$ of all orbital neoplasms [1]. These tumors usually arise from sensory nerves traversing the orbit, most frequently from the supraorbital and supratrochlear nerves. They are solitary tumors with well-defined margins [2]. Clinically, orbital schwannomas commonly present in the second to fourth

Correspondence: Kwang Seog Kim

Department of Plastic and Reconstructive Surgery, Chonnam National University Medical School, 42 Jebong-ro, Dong-gu, Gwangju 61469, Korea

E-mail: pskim@chonnam.ac.kr

Received June 2, 2015 / Revised June 24, 2015 / Accepted August 4, 2015 decade of life and tend to evolve over a period of several months to years [3]. The main symptom of orbital schwannomas is slow progressive painless ocular proptosis due to indolent nature of the growth [4]. Orbital schwannomas are usually asymptomatic when the tumors are small. However, tumor growth may cause compression to the nerve from which the tumor originated or to adjacent structures within the orbit. In this report, we describe cases of orbital schwannoma encountered in recent years.

\section{METHODS}

A retrospective review was performed to identify patients with histologically confirmed orbital schwannoma, among a list of 437 
patients who had visited our hospital with soft tissue masses within the orbit as the primary presentation between 2010 and 2014. Patient charts and medical records were reviewed for demographic information, relevant medical and family history, physical examination findings relating to ocular and extraocular sensorimotor function, operative details, postoperative complications, pathologic report, and recurrence.

\section{RESULTS}

Out of the 437 patients with orbital tumors, the review identified five patients with histologically confirmed orbital schwannoma (5/437, 1.1\%). The mean age of patients with orbital schwannoma was 47.6 years (range, 31-57 years). The mean follow-up period after treatment was 17 months (range, 5-50 months). All patients were women, and tumors were extraconal in all cases. In all cases, no pertinent medical or family history was reported for ophthalmic diseases.

Preoperative computed tomography (CT) imaging was available for all five patients. Contrast-enhanced magnetic resonance imaging (MRI) was available in two patients. In two cases, the preoperative diagnoses on CT assessment was compatible with the final histologic diagnoses. For MRI, one preoperative imaging diagnosis was compatible with postoperative final diagnosis. In all other cases, the imaging studies were not predictive of the pathologic diagnosis.

While all of the histology was that of schwannoma, one of the tumors was of Antoni type A pattern without Verocay bodies. The remaining four tumors were of mixed Antoni type A and B patterns with Verocay bodies. In all cases, immunohistochemical staining demonstrated the presence of S-100 protein, which is specific to neural crest cells.

There were no surgical complications, and postoperative ocular examination results were normal in all cases.

In two patients, postoperative numbness was noted in the area innervated by the nerve from which the tumor originated. Both of these patients reported these sensations to return, and this recovery was consistent with sensory exam at the three-month follow up visit. Postoperative scars were imperceptible, and all patients were satisfied. Clinical tumor recurrence was not noted in any of the cases. These five cases are summarized in Table 1.

\section{Case 1}

A 51-year-old woman presented with an eight-year history of a progressively enlarging mass in the right upper eyelid. She had no history of familial or ophthalmic diseases. The patient had experienced a mild headache for eight years but did not take any medication for this. On ocular examination, eyeball movements, pupillary reaction, color vision, visual fields, and neurological examination were all normal with no evidence of diplopia or proptosis. Mild vertical dystopia was present preoperatively. Preoperative orbital CT revealed a poorly enhancing mass, with a size of $20 \times 9 \times 7 \mathrm{~mm}$, in the superomedial extraconal space of the right orbit. The preoperative CT was evaluated as a benign mass such as an epidermoid cyst. The preoperative MRI diagnosis was a benign mass such as a neurogenic tumor.

Under general anesthesia, the lesion was approached via a su-

Table 1. Summary of patient characteristics

\begin{tabular}{|c|c|c|c|c|c|c|c|c|c|c|c|c|}
\hline Case & $\begin{array}{l}\text { Age } \\
(y r)\end{array}$ & Sex & Symptoms & Duration & $\begin{array}{l}\text { Size } \\
(\mathrm{mm})\end{array}$ & $\begin{array}{l}\text { Preoperative diagnosis } \\
\text { of CT scan }\end{array}$ & $\begin{array}{c}\text { Preoperative } \\
\text { diagnosis of MRI scan }\end{array}$ & Origin & $\begin{array}{l}\text { Anesthesia } \\
\text { for surgery }\end{array}$ & $\begin{array}{l}\text { Histologic } \\
\text { type }\end{array}$ & $\begin{array}{l}\text { Postoperative } \\
\text { complications }\end{array}$ & $\begin{array}{l}\text { Follow-up } \\
\text { period (mo) }\end{array}$ \\
\hline 1 & 51 & Female & $\begin{array}{l}\text { Painless palpable mass, } \\
\text { headache }\end{array}$ & $8 \mathrm{yr}$ & $20 \times 9 \times 7$ & Epidermoid cyst & Neurogenic tumor & Supraorbital nerve & General & $\begin{array}{l}\text { Antoni } \\
\text { A+Antoni B }\end{array}$ & $\begin{array}{l}\text { Temporary numbness } \\
\text { of forehead and scalp }\end{array}$ & 5 \\
\hline 2 & 31 & Female & Painless palpable mass & $5 y r^{\prime}$ & $20 \times 18 \times 16$ & $\begin{array}{l}\text { 1) Hemangioma } \\
\text { 2) Inflammatory pseudotumor } \\
\text { 3) Lymphoma }\end{array}$ & $\begin{array}{l}\text { Cavernous } \\
\text { hemangioma }\end{array}$ & $\begin{array}{l}\text { Infraorbital nerve } \\
\text { (inferior palpebral } \\
\text { branch) }\end{array}$ & General & $\begin{array}{l}\text { Antoni } \\
\text { A+Antoni B }\end{array}$ & None & 8 \\
\hline 3 & 46 & Female & Painless palpable mass & $2 \mathrm{yr}$ & $8 \times 7 \times 6$ & Neurogenic tumor & Not performed & Supratrochlear nerve & Local & Antoni A & None & 9 \\
\hline 4 & 57 & Female & Painless palpable mass & $1 \mathrm{mo}$ & $13 \times 9 \times 11$ & Schwannoma & Not performed & Supraorbital nerve & Local & $\begin{array}{l}\text { Antoni } \\
\text { AtAntoni B }\end{array}$ & $\begin{array}{l}\text { Temporary numbness } \\
\text { of forehead }\end{array}$ & 50 \\
\hline 5 & 53 & Female & Painless palpable mass & $6 \mathrm{mo}$ & $9 \times 7 \times 3$ & $\begin{array}{l}\text { 1) Benign soft tissue mass } \\
\text { 2) Inflammatory lesion }\end{array}$ & Not performed & Supratrochlear nerve & Local & $\begin{array}{l}\text { Antoni } \\
\text { A+Antoni B }\end{array}$ & None & 13 \\
\hline
\end{tabular}

CT, computed tomography; MRI, magnetic resonance imaging. 
praorbital transcutaneous incision. The mass was located in the superomedial orbit, and extended from the superior orbital foramen to the superior orbital fissure. The right supraorbital notch was widened. As the mass was well encapsulated and did not invade adjacent orbital structures, it was completely dissected away from the surrounding tissue. The supraorbital nerve was spared. Postoperative course was uneventful, with a complaint of mild paresthesia of the right forehead and scalp. No signs of recurrence or related complaints were noted in follow up (Fig. 1).

\section{Case 2}

A 31-year-old woman presented with a five-year history of a slowenlarging, palpable, and painless mass of the left lower eyelid. Medical and family history was unremarkable. On ocular examination, eyeball movements were found to be normal with no evidence of diplopia, dystopia, or proptosis. Preoperative orbital CT revealed a poorly enhancing mass, $20 \mathrm{~mm}$ in diameter, in the left inferior extraconal orbit. The preoperative CT differential diagnosis
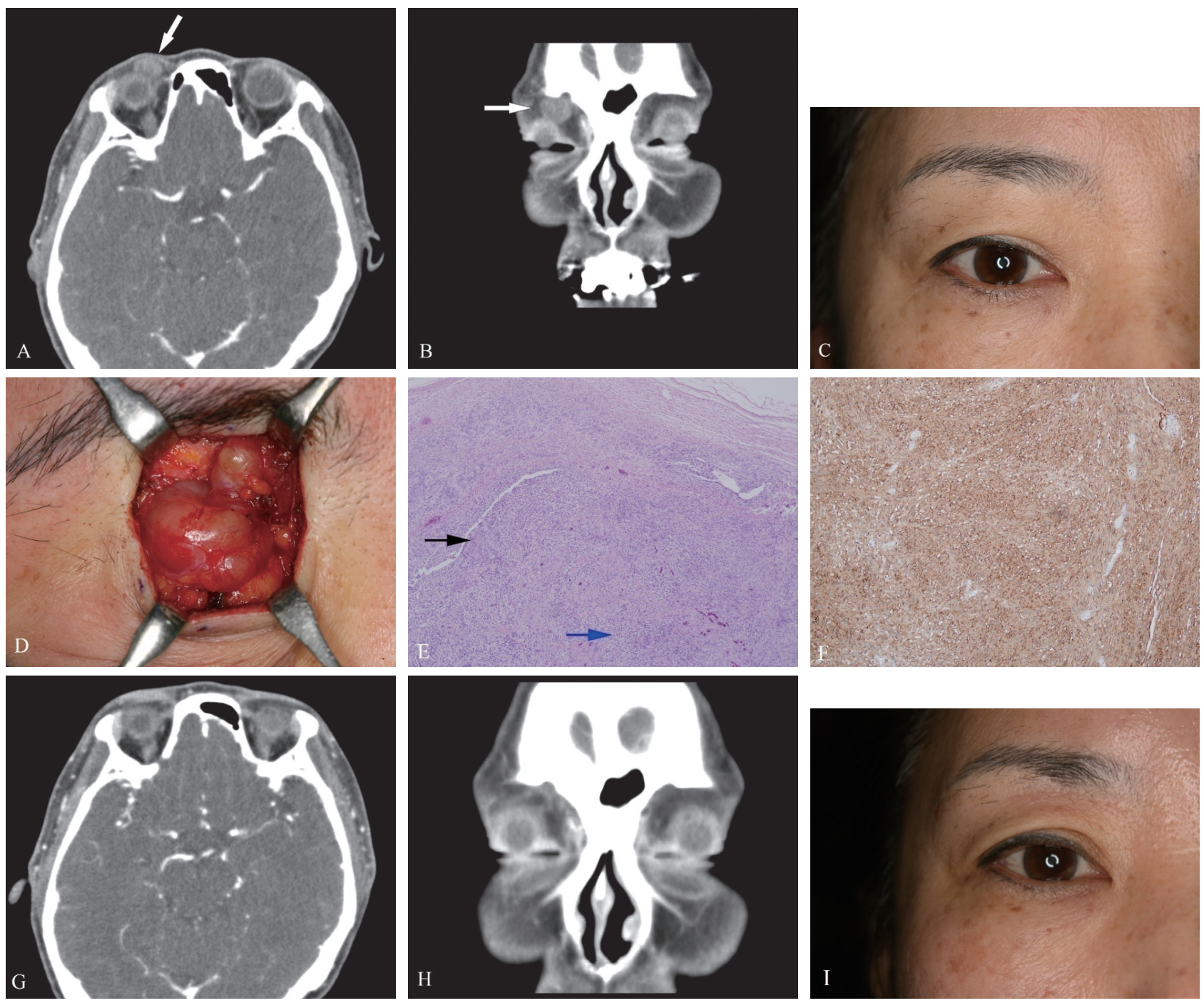

Fig. 1. Case 1. This 51-year-old woman presented with an eight-year history of a progressively enlarging mass on her right upper eyelid (white arrows). (A) Preoperative axial computed tomography (CT) image. (B) Preoperative coronal CT image. (C) Preoperative photograph. (D) Intraoperative photograph showing the mass. (E) Photomicrograph of a tumor specimen showing a biphasic pattern of Antoni A areas (black arrow) with Verocay bodies and Antoni B areas (blue arrow) (H\&E, $\times 40)$. (F) Immunohistochemical analysis showing positive staining for S-100 protein $(\mathrm{S}-100, \times 100)$. (G) Postoperative axial CT image. (H) Postoperative coronal CT image. (I) Follow-up photograph at five months. 
was hemangioma, inflammatory pseudotumor, or lymphoma. The preoperative MRI diagnosis was a cavernous hemangioma.

Under general anesthesia, surgery was performed via a left subciliary incision. The anterior portion of the mass was exposed by opening the orbital septum. The mass was found to be attached to the infraorbital nerve. The adjacent structures were delicately identified and dissected from the tumor. This firm, encapsulated mass was excised en bloc. Mild depression of the adjacent facial bones was observed. Postoperatively, the patient recovered without any complication. Final histology of the surgical specimen was consistent with schwannoma. Postoperative orbital movements were normal, and the patient was without diplopia. The eyeballs and malar surface anatomy was symmetrical, and the surgical scar was imperceptible. Facial numbness was not observed, and postoperative CT confirmed a complete removal of the tumor from the orbital space (Fig. 2).
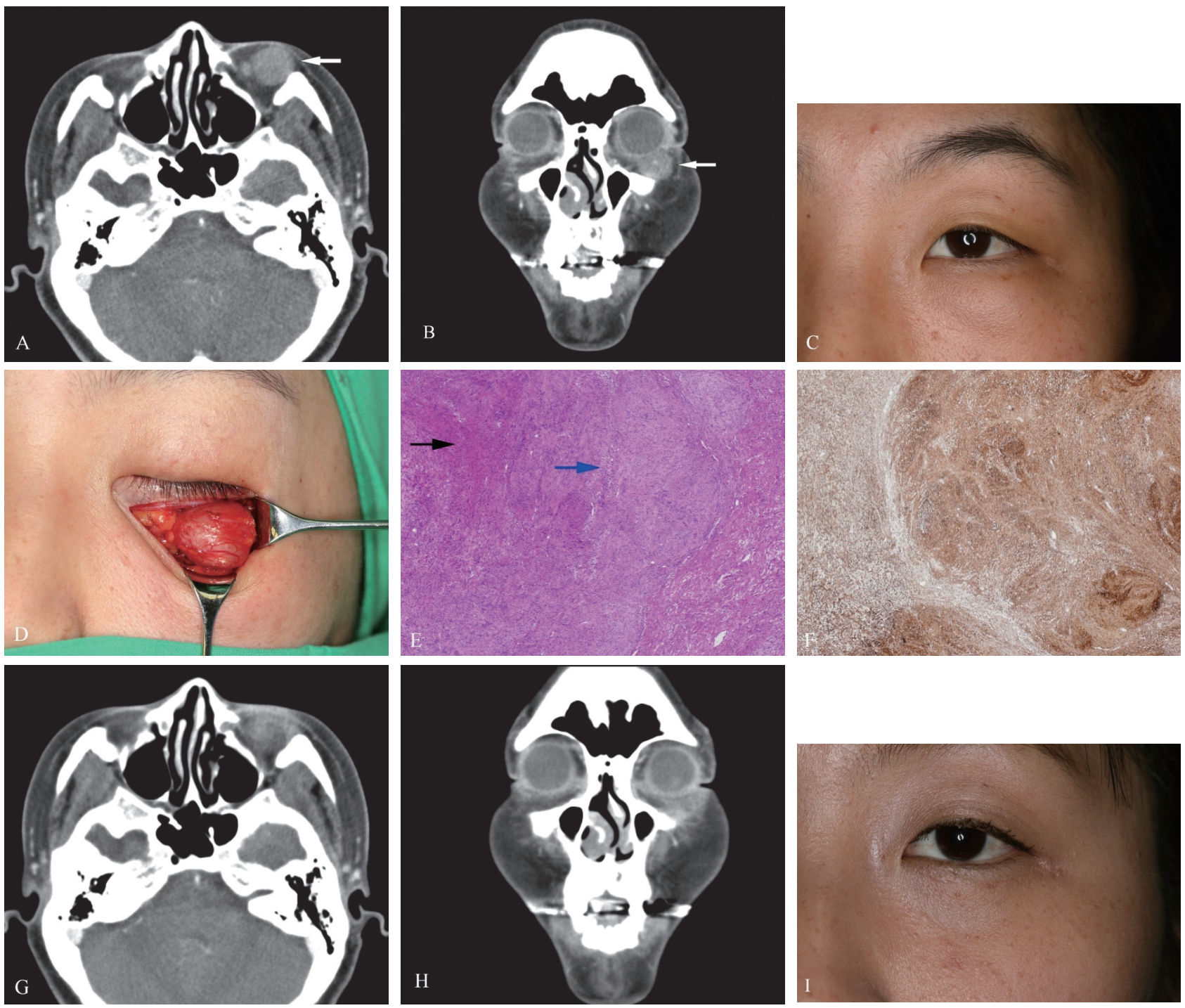

Fig. 2. Case 2. This 31-year-old woman presented with a five-year history of a slowly enlarging, palpable, and painless mass in her left lower eyelid (white arrows). (A) Preoperative axial computed tomography (CT) image. (B) Preoperative coronal CT image. (C) Preoperative photograph. (D) Intraoperative photograph showing the mass. (E) Photomicrograph of a tumor specimen showing typical manifestation of schwannoma with Antoni A areas (black arrow) containing Verocay bodies and Antoni B areas (blue arrow) (H\&E, ×40). (F) Immunohistochemical analysis showing positive staining for S-100 protein $(\mathrm{S}-100, \times 40)$. (G) Postoperative axial CT image. (H) Postoperative coronal CT image. (I) Follow-up photograph at eight months. 


\section{DISCUSSION}

As first described by Verocay in 1908, schwannomas are benign tumors, originating from Schwann cells of the peripheral nerves [5]. Orbital schwannomas are rare tumors, which generally appear in patients between 20 to 70 years of age [6]. In the orbit, specific origins of tumors often cannot be identified owing to the great complexity of the orbital structures. However, most schwannomas originate from branches of the oculomotor, trochlear, trigeminal, and abducens nerves, as well as from sympathetic and parasympathetic fibers [5]. Orbital schwannomas are mostly asymptomatic when small, but as they gradually grow, progressive painless ocular proptosis can develop and prompt patients to seek medical attention [4]. In all of our cases, there were no ocular or other functional deficits, and the chief complaint was that of a palpable mass in the orbit. Interestingly, the patient in Case 1 had been experiencing intermittent headache preoperatively. This was not examined prior to the operation. However, the patient reported that the headache had disappeared after the operation. Considering the clinical history and the temporal relationship between resection and cessation of head, the patient most likely was experiencing subclinical supraorbital neuralgia. At latest follow up, the patient continued to be free of the headache.

Most orbital schwannomas originate from sensory nerves and do not affect extraocular movements or vision, unless a tumor is located at the orbital apex oris large enough to compress the optic nerve [7]. Identifying the originating nerve is difficult because of the great number of nerve fibers traversing the orbit as well as the volume of fatty tissue located in the confined space of orbit. If a patient demonstrates functional deficit, the originating nerve can be identified in the preoperative setting [8]. In our group of asymptomatic patients, the involved nerve was discovered upon exploration. In two cases, the tumor was located in the supraorbital notch and originated from the supraorbital nerve. In two other cases, the tumor was located in the naso-orbital fossa and originated from the supratrochlear nerve. In the last remaining case, the tumor originated from the inferior palpebral branch of infraorbital nerve.

Traditionally, CT has been considered as a useful diagnostic tool, particularly in deficit-localizing lesions, to determine whether a tumor is contained within the orbit or extends into periorbital tissues or paranasal sinuses [1]. Schwannomas share similar radiographic features with cavernous hemangiomas, fibrous histiocytomas, and hemangiopericytomas. In our series, the CT diagnosis corresponded to final histologic diagnosis less than half of the time $(2 / 5,40 \%)$.

Recently, MRI has become the method of choice for examining patients with suspected orbital schwannomas because of its high sensitivity, especially with the use of contrast agents. On MRI studies, orbital schwannomas are usually described as lesions producing low-intensity signal on T1-weighted images and high-intensity signal on T2-weighted images, which can be homogeneously or heterogeneously enhanced [9]. In our case series, MRI diagnosis was consistent with final diagnosis in one of the two patients. In the "incorrect" case, the MRI was read as a cavernous hemangioma. Although we did not analyze this in our patient, Tanaka et al. [10] reported that the contrast enhancement spread pattern during MRI could be used to distinguish between cavernous hemangioma and schwannoma. Those authors proposed that physicians analyze the contrast enhancement spread pattern using dynamic MRI to achieve improved accuracy of diagnoses.

For orbital schwannomas, the surgical goal should be to completely excise the intact tumor at the earliest possible stage to prevent compression of the optic nerve [11]. Schwannomas may be successfully dissected away from the nerve of origin because of the characteristic peripheral outpouching [1]. Incomplete excision of the tumor can lead to recurrence or even intracranial extension. Highly cellular tumors have a greater chance of recurrence and malignant transformation. Therefore, early treatment is essential to prevent complications related to progressive growth of the tumor [6]. In our patients, the tumors were extraconal and the patients had presented while asymptomatic, which allowed for successful excision without any complication.

The surgical approach should be dependent on tumor location. A subciliary approach is the most preferred method for masses inferior and medial to the optic nerve. This incision provides a good operative field of view and results in excellent postoperative cosmesis [7]. In Case 2, the mass was located in the inferior extra- 
conal orbit and was accessed via a subciliary approach. If a tumor is present in both within and outside of the orbital cone, anterior orbitotomy or supra-orbitotomy approaches may be useful. If a wide operative field is necessary for sufficient dissection, lateral or transcranial orbitotomy should also be considered [12].

Grossly, schwannomas usually have the characteristic appearance of a smooth, elongated encapsulated mass. The cut tissue surface can be a relatively homogeneous greyish-white area or irregular yellow areas with cyst-like spaces. Hemorrhagic areas and thick-walled vessels can also be found [4]. A diagnosis of schwannoma can be confirmed by histopathological examination. There are two patterns of cell morphology found in schwannomas: Antoni types A and B. The Antoni type A is characterized by closely packed spindle cells having fusiform nuclei and eosinophilic cytoplasm. Schwannomas of type B pattern is characterized by haphazardly distributed cells with distinct cytoplasmic margins [11]. Nuclei of type A cells palisade to create a picket fence type structure with interdigital cytoplasmic processes, forming a pattern known as Verocay bodies [1]. Immunohistochemical analysis of schwannoma cells show positive staining for S-100 and vimentin, both of which are known to be present in Schwann cells. In all our cases, tumor cells exhibited positive staining for the S-100 protein. Staining for vimentin had not been performed.

Potential differential diagnoses for schwannomas include hemangioma, hemangiopericytoma, leiomyoma, fibrosarcoma, leiomyosarcoma, fibrous histiocytoma, solitary fibrous tumor, and meningioma [13]. At times, schwannoma presents with cystic degeneration, which can resemble dermoid cysts, mucocele, pyocele, meningoencephalocele, hematocele, epidermal inclusion cyst, and teratoma [14]. In our study, the preoperative differential on CT and MRI studies included epidermoid cyst, lymphoma, neurogenic tumor, inflammatory lesion, and cavernous hemangioma. It is difficult to diagnose schwannoma accurately owing to the variety of presentations and similarity of the orbital masses to other lesions.

In conclusion, orbital schwannomas are relatively rare tumors.
Preoperative diagnosis is difficult because of its variable presentation and location. Appropriate early assessment of orbital tumors by CT or MRI and prompt management is warranted to prevent the development of severe complications. Therefore, orbital schwannomas should be considered in the differential diagnosis of slow-growing orbital masses.

\section{REFERENCES}

1. Rootman J, Goldberg C, Robertson W. Primary orbital schwannomas. Br J Ophthalmol 1982;66:194-204.

2. Mora-Rios LE, Rios Y Valles-Valles D, Flores-Estrada JJ, RodriguezReyes AA. Infraorbital schwannoma: case report. Cir Cir 2014;82:76-80.

3. Volpe NJ, Gausas RE. Optic nerve and orbital tumors. Neurosurg Clin N Am 1999;10:699-715.

4. Lam DS, Ng JS, To KF, Abdulah V, Liew CT, Tso MO. Cystic schwannoma of the orbit. Eye (Lond) 1997;11(Pt 6):798-800.

5. Brucoli M, Giarda M, Arcuri F, Benech A. A benign isolated schwannoma of the orbit. J Craniofac Surg 2011;22:2372-4.

6. Garg R, Dhawan A, Gupta N, D'Souza P. A rare case of benign isolated schwannoma in the inferior orbit. Indian J Ophthalmol 2008;56: 514-5.

7. Tezer MS, Ozcan M, Han O, Unal A, Ozlugedik S. Schwannoma originating from the infraorbital nerve: a case report. Auris Nasus Larynx 2006;33:343-5.

8. Takahashi Y, Abe T, Ueno S, Yuge T, Maruiwa H, Tokutomi T, et al. Clinicopathological features of intraorbital neurinoma: report of two cases. Kurume Med J 1998;45:151-4.

9. Wang Y, Xiao LH. Orbital schwannomas: findings from magnetic resonance imaging in 62 cases. Eye (Lond) 2008;22:1034-9.

10. Tanaka A, Mihara F, Yoshiura T, Togao O, Kuwabara Y, Natori Y, et al. Differentiation of cavernous hemangioma from schwannoma of the orbit: a dynamic MRI study. AJR Am J Roentgenol 2004;183:1799-804.

11. Konrad EA, Thiel HJ. Schwannoma of the orbit. Ophthalmologica 1984;188:118-27.

12. Hayashi Y, Watanabe T, Kita D, Hayashi Y, Takahira M, Hamada J. Orbital cystic schwannoma originating from the frontal nerve. Case Rep Ophthalmol Med 2012;2012:604574.

13. Malone JP, Lee WJ, Levin RJ. Clinical characteristics and treatment outcome for nonvestibular schwannomas of the head and neck. Am J Otolaryngol 2005;26:108-12.

14. Tsuzuki N, Katoh H, Ohnuki A, Ishihara S, Miyazawa T, Nawashiro H, et al. Cystic schwannoma of the orbit: case report. Surg Neurol 2000; 54:385-7. 